\title{
Modelling the immunosuppressive effect of liver SBRT by simulating the dose to circulating lymphocytes: an in-silico planning study
}

\author{
L. Basler ${ }^{*}$, N. Andratschke, S. Ehrbar, M. Guckenberger and S. Tanadini-Lang
}

\begin{abstract}
Background: Tumor immune-evasion and associated failure of immunotherapy can potentially be overcome by radiotherapy, which however also has detrimental effects on tumor-infiltrating and circulating lymphocytes (CL). We therefore established a model to simulate the radiation-dose delivered to $\mathrm{CL}$.

Methods: A MATLAB-model was established to quantify the CL-dose during SBRT of liver metastases by considering the factors: hepatic blood-flow, -velocity and transition-time of individual hepatic segments, as well as probability-based recirculation. The effects of intra-hepatic tumor-location and size, fractionation and treatment planning parameters (VMAT, 3DCRT, photon-energy, dose-rate and beam-on-time) were analyzed. A threshold dose $\geq 0.5 \mathrm{~Gy}$ was considered inactivating $\mathrm{CL}$ and $\mathrm{CL} 0.5$ (\%) is the proportion of inactivated $\mathrm{CL}$.

Results: Mean liver dose was mostly influenced by treatment-modality, whereas CL0.5 was mostly influenced by beam-ontime. 3DCRT and VMAT (10MV-FFF) resulted in lowest CL0.5 values of 16 and 19\%. Metastasis location influenced CL0.5, with a mean of 19\% for both apical and basal and 31\% for the central location. PTV-volume significantly increased CL0.5 from 27 to $67 \%$ (10MV-FFF) and from 31 to $98 \%$ (6MV-FFF) for PTV-volumes ranging from $14 \mathrm{~cm}^{3}$ to $268 \mathrm{~cm}^{3}$.

Conclusion: A simulation-model was established, quantifying the strong effects of treatment-technique, tumor-location and tumor-volume on dose to $\mathrm{CL}$ with potential implications for immune-optimized treatment-planning in the future.
\end{abstract}

Keywords: Immunotherapy, Modelling, Treatment planning, Stereotactic body radiotherapy (SBRT), Abscopal effect

\section{Background}

Despite continuous multidisciplinary efforts, the prospect to transform advanced tumors into a state of "chronic disease" are still limited. Immunotherapy has shown encouraging clinical results by enhancing or inducing tumor-specific immune responses [1], however, tumor immune evasion represents a major challenge of cancer treatment today [2-4].

Major reasons for immune evasion are the immunosuppressive microenvironment of the tumor and insufficient infiltration of immune competent cells into the tumor [1, 5-11]. Radiotherapy has been demonstrated to overcome the immunosuppressive tumor microenvironment

\footnotetext{
* Correspondence: Lucas.Basler@usz.ch

University Hospital Zurich, Department of Radiation Oncology, University of Zurich, Rämistrasse 100, CH 8091 Zürich, Switzerland
}

[12] and anecdotal reports suggest that local tumor irradiation may also exert systemic or abscopal anti-tumor effects by immune-response modification with subsequent response of non-irradiated tumor metastases [13-22]. Radiation does however also have detrimental effects not only on tumor infiltrating lymphocytes but also on circulating lymphocytes (CL) in the bloodstream during radiotherapy, as these cells are particularly radiosensitive [23]. In addition, prolonged lymphopenia during or after the tumor treatment has been shown to be a prognostic factor for overall survival in many cancer types and radiotherapy may be an important factor [24-27]. In this regard, circulating lymphocytes should be treated as a radiosensitive organ at risk.

Conventional fractionated radiotherapy could have an increased negative impact compared to hypofractionated 
approaches such as SBRT, because of usually larger irradiated volumes and overall treatment times of several weeks. This might lead to a significantly larger percentage of lymphocytes receiving a dose of more than $0.5 \mathrm{~Gy}$, which is considered as a threshold dose for impaired lymphocyte function $[23,28]$. The use of stereotactic body radiotherapy (SBRT) has increased rapidly in recent years and changed the field of radiation therapy in general, as well as outcome for select patients tremendously $[29,30]$. Nearly all studies combining radiotherapy and immunotherapy focus on the use of SBRT with high ablative doses and a low number of fractions or even single fraction stereotactic radiosurgery (SRS).

This study aimed to establish a model to quantify the radiation dose delivered to $\mathrm{CL}$. The proportion of circulating lymphocytes exposed to at least 0.5 Gy (CLO.5) was used as a surrogate parameter for radiation-induced immunosuppression. The model was established for SBRT of intra-hepatic metastases because many cancer types metastasize into the liver and liver SBRT is well established in the radiation oncology community. We analyzed the influence of various SBRT planning and delivery parameters such as dose rate, beam energy, treatment time, fractionation and treatment technique on the immunosuppressive effects of radiotherapy; additionally, the influence of PTV volume and intra-hepatic tumor location were investigated.

\section{Methods}

\section{Model setting}

For this in-silico planning study, three virtual liver metastases, positioned at different intrahepatic locations, were planned with SBRT of $3 \times 15$ Gy.

A contrast-enhanced CT scan of the liver was used for treatment planning. Contouring was performed in the treatment planning software Eclipse (Varian Medical Systems). Contours included the whole liver $\left(1195 \mathrm{~cm}^{3}\right)$, the hepatic segmentation into eight individual segments (I VIII), portal vein, hepatic artery, inferior vena cava, aorta, lungs, spleen and kidneys. Contouring was performed according to the normal organ contouring guidelines for Radiation Therapy Oncology Group (RTOG) trials [31]. Three virtual liver metastases with a spherical diameter of $2 \mathrm{~cm}$ were delineated with either apical (a), basal (b) or central (c) location (Fig. 1). A PTV margin of $0.5 \mathrm{~cm}$ was added leading to a spherical diameter of $3 \mathrm{~cm}$ and a PTV volume of $14 \mathrm{~cm}^{3}$. While we kept the lesion size equal for all tumor locations and treatment modalities for comparability, we generated three additional larger lesions in the central location to evaluate volume-effects on circulating lymphocytes. Larger GTV diameters of 4, 5 or $7 \mathrm{~cm}$ were chosen resulting in PTV volumes of $65 \mathrm{~cm}^{3}, 113 \mathrm{~cm}^{3}$ and $268 \mathrm{~cm}^{3}$, respectively.

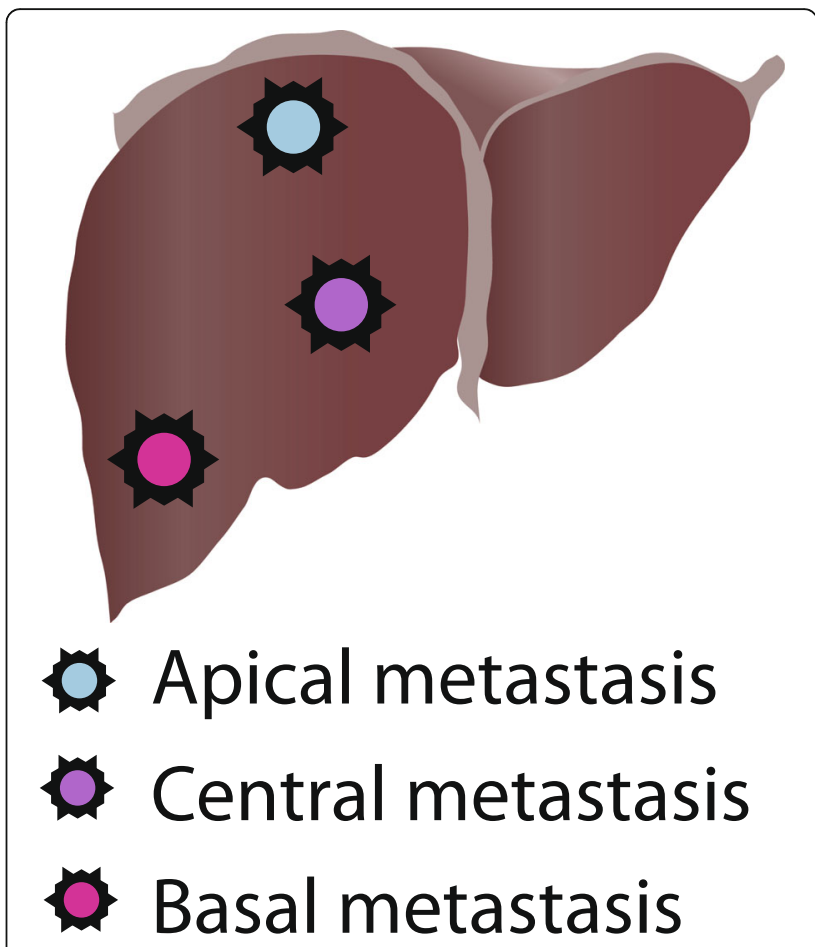

Fig. 1 Intrahepatic locations of the three virtual liver metastasis

Eclipse was used for treatment planning and DVH dose calculation. A fractionation of $3 \times 15$ Gy was chosen with an inhomogeneous dose prescription to the PTV encompassing $75 \%$ isodose line and treatment plans were normalized to a mean GTV dose of 56.25 Gy in all plans and treatment modalities. In total, 58 plans were generated using 3D conformal radiotherapy (3DCRT) and volumetric modulated arc therapy (VMAT). Details of planning characteristics are described in Table 1 . To evaluate potential fractionation effects, two additional VMAT and 3DCRT fractionation schemes were analyzed $(10 \times 4.5$ Gy and $20 \times 2.25 \mathrm{~Gy}$ ), normalized to the same mean GTV dose of $56.25 \mathrm{~Gy}$.

\section{Model description}

Cumulative dose to CLs was calculated using a liver segment specific DVH based convolution algorithm with the following assumptions:

(1) About $7-9 \%$ of the arterial and $20-23 \%$ of the intestinal bloodflow pass through the liver, which equals to $27-32 \%$ of the total cardiac output $[32,33]$. We used a value of $30 \%$ for our model.

(2)Regional hepatic blood flow is comparable in the different liver segments [34].

(3) Mean hepatic blood flow velocity is $10 \mathrm{~mm} / \mathrm{s}$.

(4) Total body blood volume is $5 \mathrm{l}$.

(5)Cardiac output is $5 \mathrm{l} / \mathrm{min}$, resulting in a circulation time of $60 \mathrm{~s}$ for the total blood volume. 
Table 1 Total beam-on time (BOT), mean liver dose (MLD) and proportion of circulating lymphocytes receiving $\geq 0.5 \mathrm{~Gy}$ (CL0.5) by treatment modality, beam energy and dose rate as a mean for all intrahepatic locations

\begin{tabular}{|c|c|c|c|c|c|c|c|}
\hline \multicolumn{8}{|c|}{ 1. Treatment modalities and mean MLD and CL0.5 of all locations } \\
\hline Treatment modality & Beam energy & Flattening filter & Dose rate $(\mathrm{MU} / \mathrm{min})$ & Fields / Arcs & Total BOT & MLD & CL0.5 \\
\hline 3DCRT & $10 \mathrm{MV}$ & FFF & 2400 & 7 & $57 \mathrm{~s}$ & $4.3 \mathrm{~Gy}$ & $15.9 \%$ \\
\hline 3DCRT & $6 \mathrm{MV}$ & FFF & 1400 & 7 & $111 \mathrm{~s}$ & $4.3 \mathrm{~Gy}$ & $23.6 \%$ \\
\hline 3DCRT & $6 \mathrm{MV}$ & FF & 600 & 7 & $253 \mathrm{~s}$ & 4.3 Gy & $30.9 \%$ \\
\hline VMAT & $10 \mathrm{MV}$ & FFF & 2400 & 2 & $74 \mathrm{~s}$ & 3.6 Gy & $19.0 \%$ \\
\hline VMAT & $6 \mathrm{MV}$ & FFF & 1400 & 3 & $147 \mathrm{~s}$ & 3.6 Gy & $23.6 \%$ \\
\hline VMAT & $6 \mathrm{MV}$ & FF & 600 & 4 & $363 \mathrm{~s}$ & 3.6 Gy & $25.1 \%$ \\
\hline
\end{tabular}

(6) Mean hepatic transition time is different in each segment and based on proximity to arterial blood supply and venous drainage. It was estimated using the distance to the geometric center of each liver segment from the arterial (hepatic artery) and venous (portal vein) blood supply and venous drainage (hepatic veins). Hepatic transition time per segment varied from 7 to $23 \mathrm{~s}$ (see Additional file 1).

(7) Same "volumes" of the blood stream were considered to be able to reenter the treatment field between different arcs/beams and treatment fractions, as the gantry rotation to the next arc or beam takes longer than the average hepatic transition and heart-to-heart circulation time.

(8)A dose of 0.5 Gy and greater was considered effective in inactivating or killing circulating lymphocytes. This dose cutoff has been chosen by previous groups working on modelling CL irradiation and is based on the intrinsic radiosensitivity of the different lymphocyte subsets [23].

(9) The probability of reentering a specific liver segment and thus treatment field, was calculated based on the percentage of cardiac output and relative volumes of the segments to generate a probabilitybased DVH convolution algorithm. As the cardiac output to the liver was defined as $30 \%$, the probability of reentering during each cycle the liver itself was $30 \%$, while the probability of reentering a specific segment varied from 2 to $22 \%$ (see Additional file 2).

We implemented an in-house developed model in MATLAB (Mathworks) to estimate the dose delivered to circulating lymphocytes based on the assumptions above.

\section{Statistical analysis}

The statistical analysis was performed in MATLAB, graphs and illustrations were generated in GraphPad Prism and Adobe Illustrator. The analysis considered absolute and relative values of all treatment modalities for each individual metastasis location, as well as mean values of CL0.5, CL1.0, CL2.0, MLD, dose rate, number of fractions and beam-on time.

\section{Results}

Mean liver dose (MLD) was mostly influenced by treatment modality: lowest values were achieved by VMAT followed by 3DCRT (Fig. 2a). In contrast, the proportion of CLs receiving $\geq 0.5 \mathrm{~Gy}$ (CL0.5) was mostly influenced by beam-on time (BOT), which is associated with beam energy and dose rate. Mean BOTs of 3DCRT were $57 \mathrm{~s}$ (10MV FFF), $111 \mathrm{~s}$ (6MV FFF) and $253 \mathrm{~s}$ (6MV FF), while total VMAT BOTs were $74 \mathrm{~s}$ (10MV FFF), $147 \mathrm{~s}$ (6MV FFF) and $363 \mathrm{~s}$ (6MV FF).

Seven-field 3DCRT using 10MV FFF beams followed by VMAT (10MV FFF) resulted in lowest mean CL0.5 values of 16 and 19\% (Fig. 2b). Treatment techniques using 6MV or $6 \mathrm{MV}$ FFF beam energy, which resulted in longer BOTs, showed substantially higher CL0.5 values with a mean of $24 \%$ or $31 \%$ for the 3DCRT and $24 \%$ or $25 \%$ for the VMAT plans, respectively (Table 1). Additionally, metastasis location influenced CL0.5, with a mean of $19 \%$ (11$25 \%)$ for the apical, $19 \%$ (13-27\%) for the basal and $31 \%$ (24-41\%) for the central location (Table 2).

We observed only a small fractionation effect for the VMAT technique (Fig. 3a). For 10MV FFF beam (central location) CL0.5 increased from 27\% (3 fractions) over 30\% (10 fractions) to $32 \%$ (20 fractions). We did not observe a fractionation effect for 3DCRT (see Additional file 3). An increase in PTV volume in the central tumor location led to a rapid increase of CL0.5 for all treatment modalities. Once the volume reached about $100 \mathrm{~cm}^{3}$, this influence reached a plateau and differences between modalities scaled linearly with a further increase in PTV volume (Fig. 3b). CL0.5 of VMAT (6MV FF) increased from $31 \%$ $\left(14 \mathrm{~cm}^{3}\right)$ to $80 \%\left(65 \mathrm{~cm}^{3}\right), 91 \%\left(113 \mathrm{~cm}^{3}\right)$ and $98 \%$ $\left(268 \mathrm{~cm}^{3}\right)$, while CL0.5 of VMAT (10MV FFF) only increased from $27 \%\left(14 \mathrm{~cm}^{3}\right)$ to $47 \%\left(65 \mathrm{~cm}^{3}\right), 55 \%$ $\left(113 \mathrm{~cm}^{3}\right)$ and $67 \%\left(268 \mathrm{~cm}^{3}\right)$, respectively.

We also calculated the proportion of CLs receiving $\geq 1$ Gy (CL1.0), as well as CLs receiving $\geq 2.0$ Gy (CL2.0). 

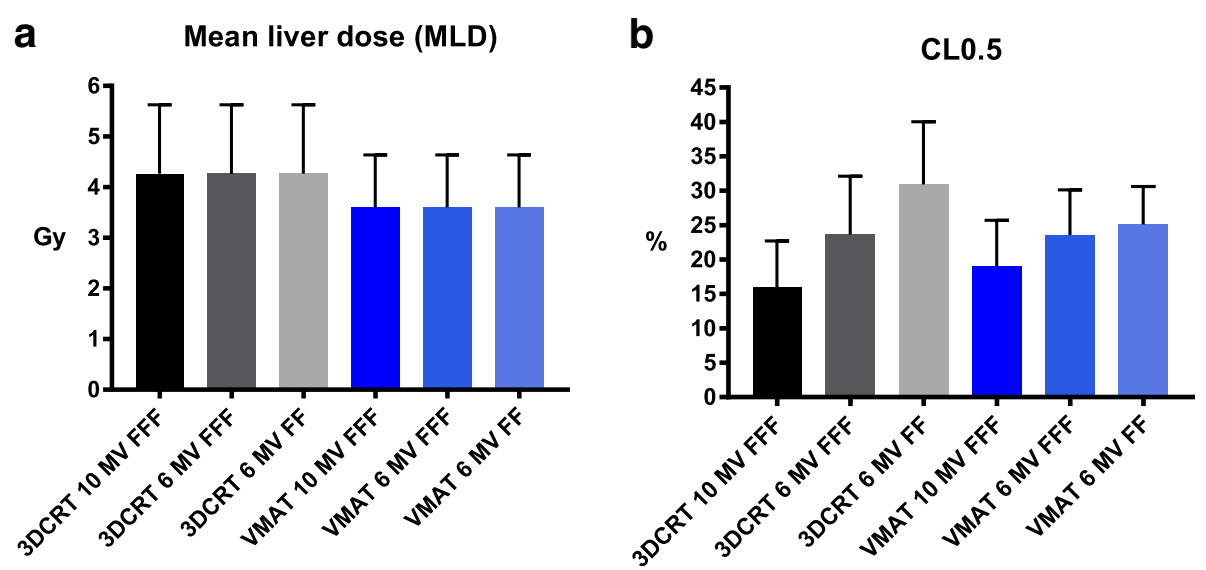

Fig. 2 Mean liver dose (MLD, a) and proportion of circulating lymphocytes receiving $\geq 0.5 G y(C L 0.5)$ by treatment modality as mean values with SD for all intrahepatic locations (b)

Higher dose rates with shorter BOTs led to increased CL1.0 and CL2.0 values, while decreasing overall CL0.5 values and general low dose exposure of CLs (Fig. 4). In larger PTV volumes, this effect was not present anymore and higher dose rates decreased overall dose to CLs, including CL0.5, CL1.0 and CL2.0 with only a slight increase of doses greater than $3 \mathrm{~Gy}$.

\section{Discussion}

Identifying predictive biomarkers for the response to immunotherapy is challenging and complex. Currently only the expression of PD-L1 is widely used clinically but there are several limitations and for many malignancies PD-L1 expression alone might be insufficient for patient selection $[35,36]$. Peripheral blood markers have been available for a long time and as previously stated: lymphopenia has been proposed as a prognostic factor for overall survival in many cancer types [24-27] and increased lymphocyte counts have also been associated with increased response and survival in the setting of immunotherapy [37-40]. It is also important to note that tumor-associated antigens released by immunogenic cell death of tumor cells are being generated during the time of radiation and have a short half-life. The timeframe around this antigen release might be most important for the induction of a systemic immune response.

Table 2 Mean liver dose (MLD) and proportion of circulating lymphocytes receiving $\geq 0.5 \mathrm{~Gy}$ (CL0.5) by intrahepatic locations as a mean for all treatment modalities and beam energies

\begin{tabular}{lll}
\hline 2. Dependence on tumor location & & \\
\hline Tumor location & MLD & CL0.5 \\
\hline Apical & $3.1 \mathrm{~Gy}$ & $18.8 \%$ \\
Basal & $3.4 \mathrm{~Gy}$ & $19.0 \%$ \\
Central & $5.3 \mathrm{~Gy}$ & $31.3 \%$ \\
\hline
\end{tabular}

Consequently, decreasing the detrimental effects of radiotherapy on circulating lymphocytes might lead to a further benefit in these scenarios.

We hypothesized that volume and location of the treated liver metastasis as well as mean liver dose would be relevant factors influencing the radiation dose delivered to circulating lymphocytes. Another assumption was that volumetric arc therapy (e.g. VMAT) with its increased spread of low dose has increased immunosuppressive effects and 3D-CRT might have advantages with decreased low dose areas and shorter beam-on times at the cost of decreased high-dose conformity.

We confirmed most of our assumptions but observed that dose rate and treatment time had a stronger impact on CL0.5 than MLD, which was mostly influenced by treatment-modality. FFF beams in both 3DCRT and VMAT drastically reduced beam-on-time resulting in significantly lower CL0.5 values compared to lower beam energies (e.g. 6MV-FF).

The Johns Hopkins and Memorial Sloan-Kettering groups have shown that SBRT had a smaller effect than conventional radiotherapy (CRT) on the total lymphocyte count (TLC) in patients with unresectable pancreatic cancer [41]. In general, patients with a higher posttreatment TLC also showed a longer survival. The Oregon Clinic group showed similar results with neoadjuvant chemoradiation for borderline resectable and locally advanced pancreatic cancer. SBRT with 30 Gy in 3 fractions over 1 week and concurrent Gemcitabine minimized treatmentassociated lymphopenia and reduced systemic loss of $\mathrm{T}$ cells, which was common in patients receiving conventional fractionated radiotherapy (CRT) with 50.4 Gy in 28 fractions over 5.5 weeks [42]. Additionally, the majority of patients receiving CRT failed to normalize their TLC posttreatment and it took up to 2 years in cases where TLC normalization occurred. Most of the SBRT patients 


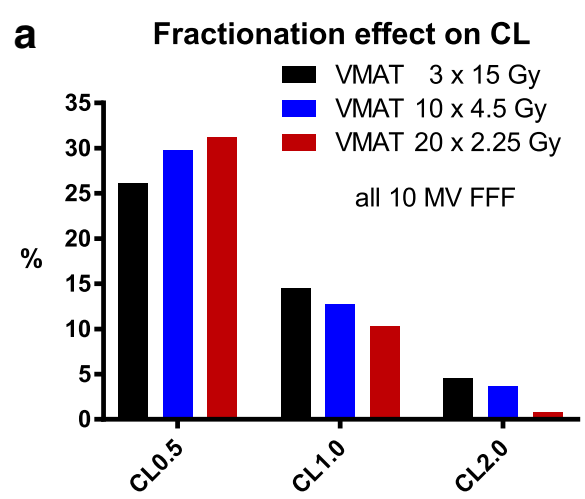

b PTV volume dependent CL0.5

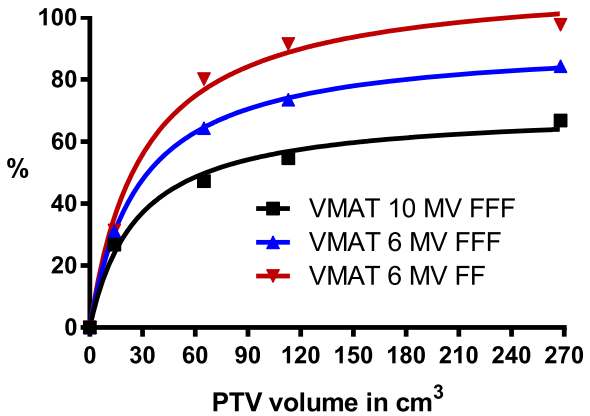

Fig. 3 Effect of either $3 \times 15$ Gy, $10 \times 4.5$ Gy or $20 \times 2.25$ Gy fractionation on CLs (a) with VMAT 10MV FFF as treatment modality. 3DCRT did not show a relevant fractionation effect (see Additional file 4). b. PTV volume dependent CL0.5 by treatment modality, based on either $14 \mathrm{~cm}^{3}, 65 \mathrm{~cm}^{3}, 114 \mathrm{~cm}^{3}$ or $268 \mathrm{~cm}^{3}$ PTV sizes in the central tumor metastasis

however where able to normalize their TLC within the treatment period.

The Johns Hopkins group showed that the total dose to circulating lymphocytes increases rapidly with the number of fractions [23]. This effect is most likely based on assumption (8) of lymphocyte re-entry, which was also part of their analysis and considers possible reentering (sub-fractions) of the cells for every field (3D-CRT) or arc (VMAT) into the treatment field. In our model, we could not see a fractionation effect with 3DCRT and noticed only a minor fractionation effect in the VMAT. Differences to previous models may be the result of our probability-based approach of each individual liver segment and implementation of differing hepatic transition times compared to the single OAR approach of Yovino et al. In contrast to the results of Yovino et al., BOT and dose rate seem to be very important factors. We used higher dose rates with up to $2400 \mathrm{MU} / \mathrm{min}$ without flattening filter, which might explain some of the differences. With higher dose rates, less circulating lymphocytes are irradiated and a smaller portion of lymphocytes receives a higher dose, as shown in Fig. 4.

In the studies of Wild and Crocenzi [41, 42], PTV size was significantly larger for the conventionally fractionated radiotherapy $\left(89 \mathrm{~cm}^{3}\right.$ SBRT vs. $345 \mathrm{~cm}^{3}$ CRT in the first and approximately $150 \mathrm{~cm}^{3}$ vs. $400 \mathrm{~cm}^{3}$ in the second study). Thus, we built a model based on equal PTV volumes, while trying to optimize intra-fractional lymphocyte sparing by choice of treatment modality, dose rate and treatment time. PTV volume showed substantially larger differences than fractionation in our analysis, suggesting that the effect described in the studies above might be volume rather than fractionation related, though this would have to be confirmed in future in-vivo experiments.

PTV volume and treatment time seem to be the most critical factors of lymphocyte sparing. In summary, immunosuppressive effects of SBRT might be minimized by avoiding treatment of large metastases, a high number of fractions and especially long treatment times in the setting of immunotherapy.

\section{CL0.0 to CL5.0}

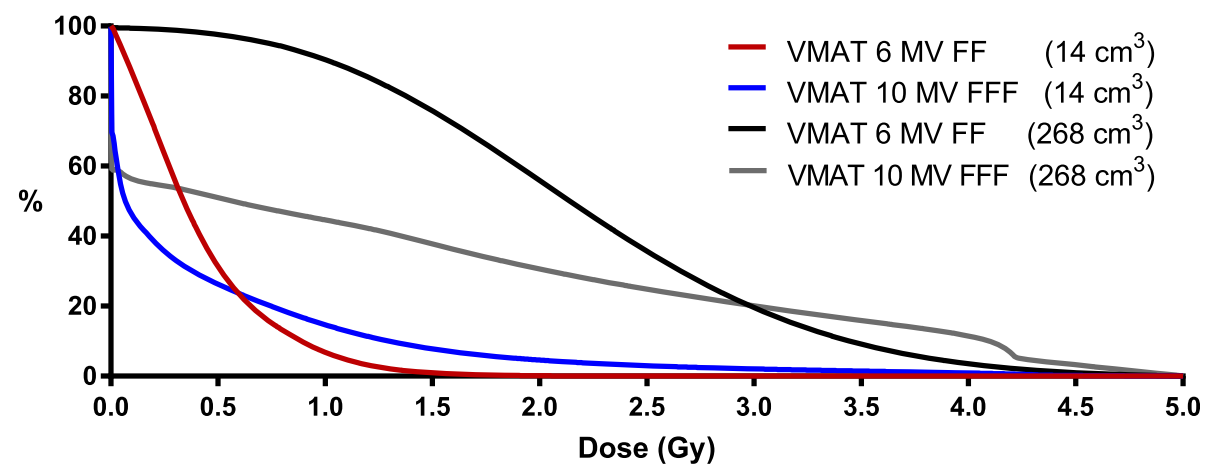

Fig. 4 CL0.0 to CL5.0 for VMAT 10MV FFF and 6MV FF in the central tumor location and either $14 \mathrm{~cm}^{3} \mathrm{or}_{268} \mathrm{~cm}^{3} \mathrm{PTV}$ volume 
There are limitations to our model, including that the value of CL0.5 is based on lymphocyte radio-sensitivity which has been assessed in vitro via colony formation assay [28] and in vivo data on dose-dependent activity and function of circulating lymphocytes is scarce [43-46]. As seen in Fig. 4, we are however able to minimize not only CL0.5 but also overall radiation exposure of CLs with the right treatment modality. Additionally, radiation sensitivity of lymphocytes is highly dependent on the specific subset. Our model is of most relevance to naïve T-cells, which are especially radiosensitive, compared to other subsets such as memory cells [47-49]. In addition, overall survival may be associated with a certain lymphocyte subset [50] and also be dependent on other cell types, e.g. innate immune cells such as neutrophils as discussed by Son et al. [51]. Regeneration and redistribution of lymphocytes, as well as local/non-circulating lymphocytes were not considered in our model and might influence the total amount of irradiated lymphocytes. This could also be dependent on tumor location and entity. However, this would likely affect all treatment modalities and inclusion of these factors would lead to a very complex model for which several parameters are unknown. Additionally, we do not know about the real impact of circulating lymphocytes on the efficacy of immunotherapy and immune-modulating antibodies may have additional influences on lymphocytes, including alteration and differentiation of subsets, as well as radiation sensitivity. However, the dependency of increased survival on absolute lymphocyte count suggests that we may be able to reduce detrimental radiation effects in this regard. The development of models for clinical decision-making is necessary for personalized treatment strategies in the metastatic situation [52]. These models should, however, be based on multiple biological and physiological parameters validated in-vivo prior to clinical application [53].

\section{Conclusion}

A simulation-model has been established to estimate and quantify potential immunosuppressive effects of radiotherapy through inactivation of circulating lymphocytes. From a technical perspective, treatment delivery time had the strongest impact on the proportion of lymphocytes receiving $\geq 0.5 \mathrm{~Gy}$ : best $\mathrm{CL}$-sparing was achieved with 10MV FFF 3DCRT followed by VMAT. However, the clinical parameters metastasis location and in particular metastasis volume had the strongest impact on the immunosuppressive effects of radiotherapy. We therefore believe that these results will be relevant in the setting of combined radio-immunotherapy with potential implications for immune-optimized treatment planning in the future.

\section{Additional files}

Additional file 1: Segment volume. Volume and blood flow per segment. Calculated absolute and relative volume and blood flow of the individual liver segments. (PDF $129 \mathrm{~kb}$ )

Additional file 2: Segment distance. Distance to geometric center and mean hepatic transition time per segment. 2D Distance from arterial blood supply/venous drainage to geometric center of individual segments. 3D Pythagorean distance calculation for estimation of mean hepatic transition time per segment (in seconds). (PDF 148 kb)

Additional file 3: DVH convulution algorithm. DVH convolution algorithm. For every treatment fraction, the current Blood DVH is multiplied by a new convolution DVH consisting of individual liver segments \& the blood fraction outside the liver. As a result, a new Blood DVH is generated. (PDF $726 \mathrm{~kb}$ )

Additional file 4: Fractionation effect on $\mathrm{CL}$ (3D CRT). Fractionation effect on $\mathrm{CL}$ (3DCRT). There is no significant fractionation effect on circulating lymphocytes with 3DCRT for the apical tumor location. (PDF 26 kb)

\section{Abbreviations}

BOT: Beam-on time; CL: Circulating lymphocytes; CL0.5: Proportion of circulating lymphocytes receiving $\geq 0.5 \mathrm{~Gy}$; CRT: Conventional radiotherapy; MLD: Mean liver dose; SBRT: Stereotactic body radiotherapy; TLC: Total lymphocyte count; VMAT: Volumetric arc therapy

\section{Acknowledgements \\ None. \\ Funding \\ No funding was received.}

Availability of data and materials

The datasets used and/or analyzed during the current study are available from the corresponding author on reasonable request.

\section{Authors' contributions}

LB was responsible for contouring, analyzing the data and writing the manuscript. STL and SE created the model for dose estimation to circulating lymphocytes. NA, MG and STL supervised the study and contributed to data interpretation. All authors read and approved the final manuscript.

Ethics approval and consent to participate

Not applicable

\section{Consent for publication}

Not applicable

\section{Competing interests}

The authors declare that they have no competing interests.

\section{Publisher's Note}

Springer Nature remains neutral with regard to jurisdictional claims in published maps and institutional affiliations.

Received: 3 August 2017 Accepted: 3 January 2018

Published online: 22 January 2018

References

1. Whiteside TL, Demaria S, Rodriguez-Ruiz ME, Zarour HM, Melero I. Emerging opportunities and challenges in cancer immunotherapy. Clin Cancer Res. 2016;22:1845-55.

2. Sporn MB. The war on cancer. Lancet. 1996;347:1377-81.

3. Sporn MB. The war on cancer: a review. Ann N Y Acad Sci. 1997;833:137-46.

4. Geiger TR, Peeper DS. Metastasis mechanisms. Biochim Biophys Acta. 2009; 1796:293-308

5. Banchereau J, Briere F, Caux C, Davoust J, Lebecque S, Liu YJ, Pulendran B, Palucka K. Immunobiology of dendritic cells. Annu Rev Immunol. 2000;18:767-811. 
6. Vicari AP, Caux C, Trinchieri G. Tumour escape from immune surveillance through dendritic cell inactivation. Semin Cancer Biol. 2002;12:33-42.

7. Gabrilovich DI, Chen HL, Girgis KR, Cunningham HT, Meny GM, Nadaf S, Kavanaugh D, Carbone DP. Production of vascular endothelial growth factor by human tumors inhibits the functional maturation of dendritic cells. Nat Med. 1996;2:1096-103.

8. Kobie JJ, Wu RS, Kurt RA, Lou S, Adelman MK, Whitesell LJ, Ramanathapuram LV, Arteaga CL, Akporiaye ET. Transforming growth factor beta inhibits the antigen-presenting functions and antitumor activity of dendritic cell vaccines. Cancer Res. 2003;63:1860-4.

9. Melief CJ. Cancer immunotherapy by dendritic cells. Immunity. 2008;29:372-83.

10. Wang HY, Wang RF. Regulatory T cells and cancer. Curr Opin Immunol. 2007:19:217-23.

11. Triozzi PL, Khurram R, Aldrich WA, Walker MJ, Kim JA, Jaynes S. Intratumoral injection of dendritic cells derived in vitro in patients with metastatic cancer. Cancer. 2000;89:2646-54.

12. Demaria S, Ng B, Devitt ML, Babb JS, Kawashima N, Liebes L, Formenti SC. lonizing radiation inhibition of distant untreated tumors (abscopal effect) is immune mediated. Int J Radiat Oncol Biol Phys. 2004;58:862-70.

13. Bhattacharyya T, Purushothaman K, Puthiyottil SS, Bhattacharjee A, Muttah G. Immunological interactions in radiotherapy-opening a new window of opportunity. Ann Transl Med. 2016;4:51.

14. Chandra RA, Wilhite TJ, Balboni TA, Alexander BM, Spektor A, Ott PA, Ng AK, Hodi FS, Schoenfeld JD. A systematic evaluation of abscopal responses following radiotherapy in patients with metastatic melanoma treated with ipilimumab. Oncoimmunology. 2015;4:e1046028.

15. Postow MA, Callahan MK, Barker CA, Yamada Y, Yuan J, Kitano S, Mu Z, Rasalan T, Adamow M, Ritter E, et al. Immunologic correlates of the abscopal effect in a patient with melanoma. N Engl J Med. 2012;366:925-31.

16. Frey B, Rubner Y, Wunderlich R, Weiss EM, Pockley AG, Fietkau R, Gaipl US. Induction of abscopal anti-tumor immunity and immunogenic tumor cell death by ionizing irradiation - implications for cancer therapies. Curr Med Chem. 2012;19:1751-64.

17. Golden EB, Chhabra A, Chachoua A, Adams S, Donach M, Fenton-Kerimian M, Friedman K, Ponzo F, Babb JS, Goldberg J, et al. Local radiotherapy and granulocyte-macrophage colony-stimulating factor to generate abscopal responses in patients with metastatic solid tumours: a proof-of-principle trial. Lancet Oncol. 2015;16:795-803.

18. Golden EB, Demaria S, Schiff PB, Chachoua A, Formenti SC. An abscopal response to radiation and ipilimumab in a patient with metastatic nonsmall cell lung cancer. Cancer Immunol Res. 2013;1:365-72.

19. Demaria S, Pilones KA, Vanpouille-Box C, Golden EB, Formenti SC. The optimal partnership of radiation and immunotherapy: from preclinical studies to clinical translation. Radiat Res. 2014;182:170-81.

20. Siva S, MacManus MP, Martin RF, Martin OA. Abscopal effects of radiation therapy: a clinical review for the radiobiologist. Cancer Lett. 2015;356:82-90.

21. Grimaldi AM, Simeone E, Giannarelli D, Muto P, Falivene S, Borzillo V, Giugliano FM, Sandomenico F, Petrillo A, Curvietto M, et al. Abscopal effects of radiotherapy on advanced melanoma patients who progressed after ipilimumab immunotherapy. Oncoimmunology. 2014;3:e28780.

22. Reynders K, Illidge T, Siva S, Chang JY, De Ruysscher D. The abscopal effect of local radiotherapy: using immunotherapy to make a rare event clinically relevant. Cancer Treat Rev. 2015;41:503-10.

23. Yovino S, Kleinberg L, Grossman SA, Narayanan M, Ford E. The etiology of treatment-related lymphopenia in patients with malignant gliomas: modeling radiation dose to circulating lymphocytes explains clinical observations and suggests methods of modifying the impact of radiation on immune cells. Cancer Investig. 2013;31:140-4.

24. Ray-Coquard I, Cropet C, Van Glabbeke M, Sebban C, Le Cesne A, Judson I, Tredan O, Verweij J, Biron P, Labidi I, et al. Lymphopenia as a prognostic factor for overall survival in advanced carcinomas, sarcomas, and lymphomas. Cancer Res. 2009;69:5383-91.

25. Campian JL, Sarai G, Ye X, Marur S, Grossman SA. Association between severe treatment-related lymphopenia and progression-free survival in patients with newly diagnosed squamous cell head and neck cancer. Head Neck. 2014:36:1747-53.

26. Wild AT, Ye X, Ellsworth SG, Smith JA, Narang AK, Garg T, Campian J, Laheru DA, Zheng $L$, Wolfgang $C L$, et al. The association between chemoradiation-related lymphopenia and clinical outcomes in patients with locally advanced pancreatic adenocarcinoma. Am J Clin Oncol. 2015;38:259-65.
27. Grossman SA, Ellsworth S, Campian J, Wild AT, Herman JM, Laheru D, Brock M, Balmanoukian A, Ye X. Survival in patients with severe lymphopenia following treatment with radiation and chemotherapy for newly diagnosed solid tumors. J Natl Compr Cancer Netw. 2015;13:1225-31.

28. Nakamura N, Kusunoki Y, Akiyama M. Radiosensitivity of CD4 or CD8 positive human T-lymphocytes by an in vitro colony formation assay. Radiat Res. 1990;123:224-7.

29. Baumann M, Krause M, Overgaard J, Debus J, Bentzen SM, Daartz J, Richter C, Zips D, Bortfeld T. Radiation oncology in the era of precision medicine. Nat Rev Cancer. 2016;16:234-49.

30. Rieber J, Streblow J, Uhlmann L, Flentje M, Duma M, Ernst I, Blanck O, Wittig A, Boda-Heggemann J, Krempien R, et al. Stereotactic body radiotherapy (SBRT) for medically inoperable lung metastases-a pooled analysis of the German working group "stereotactic radiotherapy". Lung Cancer. 2016;97:51-8.

31. Jabbour SK, Hashem SA, Bosch W, Kim TK, Finkelstein SE, Anderson BM, Ben-Josef E, Crane CH, Goodman KA, Haddock MG, et al. Upper abdominal normal organ contouring guidelines and atlas: a radiation therapy oncology group consensus. Pract Radiat Oncol. 2014;4:82-9.

32. Rowell LB. Human cardiovascular control. New York: Oxford University Press; 1993.

33. Lautt WW, Greenway CV. Conceptual review of the hepatic vascular bed. Hepatology. 1987;7:952-63.

34. Taniguchi H, Oguro A, Takeuchi K, Miyata K, Takahashi T, Inaba T, Nakahashi H. Difference in regional hepatic blood flow in liver segments-non-invasive measurement of regional hepatic arterial and portal blood flow in human by positron emission tomography with H2(15)O. Ann Nucl Med. 1993;7:141-5.

35. McLaughlin J, Han G, Schalper KA, Carvajal-Hausdorf D, Pelekanou V, Rehman J, Velcheti V, Herbst R, LoRusso P, Rimm DL. Quantitative assessment of the heterogeneity of PD-L1 expression in non-small-cell lung cancer. JAMA Oncol. 2016;2:46-54.

36. Ilie M, Long-Mira E, Bence C, Butori C, Lassalle S, Bouhlel L, Fazzalari L, Zahaf K, Lalvee S, Washetine K, et al. Comparative study of the PD-L1 status between surgically resected specimens and matched biopsies of NSCLC patients reveal major discordances: a potential issue for anti-PD-L1 therapeutic strategies. Ann Oncol. 2016;27:147-53.

37. GY K, Yuan J, Page DB, Schroeder SE, Panageas KS, Carvajal RD, Chapman PB, Schwartz GK, Allison JP, Wolchok JD. Single-institution experience with ipilimumab in advanced melanoma patients in the compassionate use setting: lymphocyte count after 2 doses correlates with survival. Cancer. 2010;116:1767-75

38. Delyon J, Mateus C, Lefeuvre D, Lanoy E, Zitvogel L, Chaput N, Roy S, Eggermont AM, Routier E, Robert C. Experience in daily practice with ipilimumab for the treatment of patients with metastatic melanoma: an early increase in lymphocyte and eosinophil counts is associated with improved survival. Ann Oncol. 2013;24:1697-703.

39. Simeone E, Gentilcore G, Giannarelli D, Grimaldi AM, Caraco C, Curvietto M, Esposito A, Paone M, Palla M, Cavalcanti E, et al. Immunological and biological changes during ipilimumab treatment and their potential correlation with clinical response and survival in patients with advanced melanoma. Cancer Immunol Immunother. 2014:63:675-83.

40. Gibney GT, Weiner LM, Atkins MB. Predictive biomarkers for checkpoint inhibitor-based immunotherapy. Lancet Oncol. 2016;17:e542-51.

41. Wild AT, Herman JM, Dholakia AS, Moningi S, Lu Y, Rosati LM, Hacker-Prietz A, Assadi RK, Saeed AM, Pawlik TM, et al. Lymphocyte-sparing effect of stereotactic body radiation therapy in patients with unresectable pancreatic cancer. Int J Radiat Oncol Biol Phys. 2016;94:571-9.

42. Crocenzi T, Cottam B, Newell P, Wolf RF, Hansen PD, Hammill C, Solhjem MC, To YY, Greathouse A, Tormoen G, et al. A hypofractionated radiation regimen avoids the lymphopenia associated with neoadjuvant chemoradiation therapy of borderline resectable and locally advanced pancreatic adenocarcinoma. J Immunother Cancer. 2016:4:45.

43. Bogdandi EN, Balogh A, Felgyinszki N, Szatmari T, Persa E, Hildebrandt G, Safrany G, Lumniczky K. Effects of low-dose radiation on the immune system of mice after total-body irradiation. Radiat Res. 2010;174:480-9.

44. Pecaut M, Nelson GA, Gridley DS. Dose and dose rate effects of whole-body gamma-irradiation: I. Lymphocytes and lymphoid organs. In Vivo. 2001;15:195-208.

45. Anderson RE, Williams WL. Radiosensitivity of T and B lymphocytes. V. Effects of whole-body irradiation on numbers of recirculating $T$ cells and sensitization to primary skin grafts in mice. Am J Pathol. 1977:89:367-78.

46. Manda K, Glasow A, Paape D, Hildebrandt G. Effects of ionizing radiation on the immune system with special emphasis on the interaction of dendritic and T cells. Front Oncol. 2012;2:102. 
47. Li HH, Wang YW, Chen R, Zhou B, Ashwell JD, Fornace AJ Jr. lonizing radiation impairs $T$ cell activation by affecting metabolic reprogramming. Int J Biol Sci. 2015;11:726-36.

48. Belka C, Ottinger H, Kreuzfelder E, Weinmann M, Lindemann M, LeppleWienhues A, Budach W, Grosse-Wilde H, Bamberg M. Impact of localized radiotherapy on blood immune cells counts and function in humans. Radiother Oncol. 1999:50:199-204.

49. Uzawa A, Suzuki G, Nakata Y, Akashi M, Ohyama H, Akanuma A. Radiosensitivity of CD45RO+ memory and CD45RO- naive T cells in culture. Radiat Res. 1994;137:25-33.

50. Grossman SA, Ye X, Lesser G, Sloan A, Carraway H, Desideri S, Piantadosi S, Consortium NC. Immunosuppression in patients with high-grade gliomas treated with radiation and temozolomide. Clin Cancer Res. 2011;17:5473-80.

51. Son SH, Park EY, Park HH, Kay CS, Jang HS. Pre-radiotherapy neutrophil-tolymphocyte ratio as an independent prognostic factor in patients with locally advanced hepatocellular carcinoma treated with radiotherapy. Oncotarget. 2017:8:16964-71.

52. Poleszczuk JT, Luddy KA, Prokopiou S, Robertson-Tessi M, Moros EG, Fishman M, Djeu JY, Finkelstein SE, Enderling H. Abscopal benefits of localized radiotherapy depend on activated T-cell trafficking and distribution between metastatic lesions. Cancer Res. 2016;76:1009-18.

53. Demaria S, Formenti SC. Can abscopal effects of local radiotherapy be predicted by modeling T cell trafficking? J Immunother Cancer. 2016;4:29.

\section{Submit your next manuscript to BioMed Central} and we will help you at every step:

- We accept pre-submission inquiries

- Our selector tool helps you to find the most relevant journal

- We provide round the clock customer support

- Convenient online submission

- Thorough peer review

- Inclusion in PubMed and all major indexing services

- Maximum visibility for your research

Submit your manuscript at www.biomedcentral.com/submit 